\title{
Discrete Cubic Interpolatory Splines
}

\author{
By \\ Manjulata SHRIVASTAVA*
}

\begin{abstract}
In the present paper, existence, uniqueness and convergence properties of a discrete cubic spline which satisfies certain averaging interpolatory condition are established. This type of interpolatory condition has been studies earlier for usual cubic splines in [3]. More precise range for weights involved in the interpolatory condition and sharper error estimates than in [3], are obtained in the present paper.
\end{abstract}

\section{§1. Introduction}

Discrete splines are piecewise polynomial functions which satisfy smoothness requirements at the knots in terms of differences. They depend for their definition, on a real parameter $h>0$. In particular, when $h \rightarrow 0$, discrete splines reduce to corresponding usual splines. Thus discrete splines are a natural generalization of usual splines. Discrete splines were introduced by Mangasarian and Schumaker [8] in connection with solution of certain minimization problems involving differences. Schumaker [9], [10], Lyche [6], [7] and Astor and Duris [2] have studied in details, different aspects of discrete spline approximation. Recently, Dikshit and Powar [4], [5] have considered different important interpolatory properties of discrete cubic splines. Our aim in this paper is to study the existence and convergence properties of discrete cubic spline which satisfies certain averaging interpolatory condition. Such an interpolatory condition has been considered by Chatterjee and Dikshit [3] for usual cubic splines. Error estimates obtained in the present paper are sharper than those obtained in [3]. Moreover, the range of variation of weight $\alpha$ involved in the interpolatory condition is determined more precisely in the present paper.

In Section 2, we shall establish the existence and uniqueness of a discrete cubic spline satisfying interpolatory condition mentioned above, while the convergence properties of these interpolatory splines are discussed in Séction 3.

Communicated by T. Kawai, December 2, 1991. Revised January 24, 1992.

1991 Mathematics Subject Classification: Primary 41 (AO5), 65 (DO7)

* Department of Mathematics and Computer Science, R.D. University, Jabalpur, 482001-India. 


\section{§2. Existence and Uniqueness}

Let a real number $h>0$ be given. Then for any fixed real number $a$, discrete real line $R_{a h}$ is given by

$$
\mathbb{R}_{a h}=\{\ldots, a-h, a, a+h, a+2 h, \ldots\}
$$

and a discrete interval $[a, b]_{h}$ is defined by

$$
[a, b]_{h}=[a, b] \cap R_{a h} .
$$

Discrete intervals $(a, b]_{h},(a, b)_{h}$ and $[a, b)_{h}$ are defined analogously.

Let $P=\left\{x_{i}\right\}_{i=0}^{n}$ such that $0=x_{0}<x_{1}<\cdots<x_{n}=1$, be a uniform sequence of points in the discrete interval $[0,1]_{h}$. Let $x_{i}-x_{i-1}=p, i=$ $1,2, \ldots, n$.

A discrete cubic spline $s$ is a piecewise cubic polynomial with knots in $P$ which satisfies following conditions

$$
\mathbb{D}_{h}^{\{j\}} S_{i}\left(x_{i}\right)=D_{h}^{\{j\}} S_{i+1}\left(x_{i}\right), \quad j=0,1 \text { and } 2, i=1,2, \ldots, n-1 ;
$$

where $s_{i}$ is the restriction of $s$ in interval $\left[x_{i-1}, x_{i}\right]$ for each $i$. Also, $D_{h}^{\{j\}} g$ denotes the $j$-th central difference of function $g$. More precisely we have,

$$
\begin{aligned}
& D_{h}^{\{0\}} g(x)=g(x), \\
& D_{h}^{\{1\}} g(x)=[g(x+h)-g(x-h)] / 2 h
\end{aligned}
$$

and

$$
D_{h}^{\{2\}} g(x)=[g(x+h)+g(x-h)-2 g(x)] / h^{2} .
$$

For convenience we shall denote $D_{h}^{\{r\}} g$ by $g^{\{r\}}$ and $g\left(x_{i}\right)$ by $g_{i}$ for any function $g$ defined on $[0,1]_{h}$. The space of discrete cubic splines with knots in $P$ is denoted by $\mathscr{S}(4, \mathbb{P}, h)$.

We shall investigate the following:

Problem 2.1. Given a 1-periodic function $f$ defined over $[0,1]_{h}$ and some non-negative number $\alpha$, does there exist a unique 1-periodic discrete cubic spline $s$ in $\mathscr{S}(4, P, h)$ satisfying the interpolatory condition

$$
s_{i-1}+\alpha s_{i}=f_{i-1}+\alpha f_{i}, \quad i=1,2, \ldots, n ?
$$

It is clear that $D_{h}^{\{2\}} s(x)$ is linear in each interval $\left[x_{i-1}, x_{i}\right]$. Therefore we have

$$
p D_{h}^{\{2\}} s(x)=\left(x_{i}-x\right) M_{i-1}+\left(x-x_{i-1}\right) M_{i}
$$

where $M_{i}=D_{h}^{\{2\}} s\left(x_{i}\right)$. Thus summing twice we get

$$
\begin{aligned}
6 p s(x)= & M_{i-1}\left(x_{i}-x\right)^{\{3\}}+M_{i}\left(x-x_{i-1}\right)^{\{3\}}+6 c_{i}\left(x_{i}-x\right) \\
& +6 d_{i}\left(x-x_{i-1}\right), \quad i=1,2, \ldots, n
\end{aligned}
$$


where $c_{i}$ and $d_{i}$ are arbitrary constants and $x^{\{j\}}$ are the factorial functions given by

$$
\begin{aligned}
x^{\{0\}} & =1, \\
x^{\{2 k\}} & =x^{2}\left(x^{2}-h^{2}\right) \ldots\left(x^{2}-(k-1)^{2} h^{2}\right), \\
x^{\{2 k-1\}} & =x\left(x^{2}-h^{2}\right) \ldots\left(x^{2}-(k-1)^{2} h^{2}\right),
\end{aligned}
$$

$k$ being a positive integer.

For given functional values $z_{i}$ on $\left[x_{i-1}, x_{i}\right]$ and real number $r$ we set

$$
\theta_{r}\left(z_{i}\right)=z_{i-1}+r z_{i}, \quad i=1, \ldots, n .
$$

It is easy to see that requirements (2.1) lead to following relations:

$$
d_{i}=c_{i+1}
$$

and

$$
p^{2} M_{i}=\theta_{-1}\left(d_{i}\right)-\theta_{-1}\left(d_{i+1}\right) \text {. }
$$

Since $s$ satisfies the interpolatory conditions (2.2) we get

$$
\left(p^{2}-h^{2}\right) \theta_{\alpha}\left(M_{i}\right) / 6+\theta_{\alpha}\left(d_{i}\right)=\theta_{\alpha}\left(f_{i}\right) \text {. }
$$

Eliminating $c_{i}$ and $d_{i}$ in (2.5)-(2.7), we get, in view of periodicity of $f$ and $s$, the following system of equations in unknowns $M_{i}, i=1, \ldots, n$ :

$$
\begin{aligned}
& \left(p^{2}-h^{2}\right) \theta_{\alpha}\left(M_{i}\right)+\left(4 p^{2}+2 h^{2}\right) \theta_{\alpha}\left(M_{i+1}\right)+\left(p^{2}-h^{2}\right) \theta_{\alpha}\left(M_{i+2}\right) \\
& \quad=6\left[\theta_{\alpha}\left(f_{i}\right)-2 \theta_{\alpha}\left(f_{i+1}\right)+\theta_{\alpha}\left(f_{i+2}\right)\right]=F_{i} \quad \text { (say), } \quad i=1,2, \ldots, n ;
\end{aligned}
$$

where $f_{n+r}=f_{r}$ and $M_{n+r}=M_{r}, r=0,1,2, \ldots$.

It is easy to observe that the system of equations (2.8) can be written in matrix-form as $A M=F$ where $M={ }^{t}\left(M_{1}, M_{2}, \ldots, M_{n}\right), F={ }^{t}\left(F_{1}, F_{2}, \ldots, F_{n}\right)$ and matrix $A$ is given by

$$
A=\left[\begin{array}{cccccccc}
y+\alpha \beta & \beta+\alpha y & \alpha \beta & 0 & 0 & \ldots & 0 & \beta \\
\beta & y+\alpha \beta & \beta+\alpha y & \alpha \beta & 0 & \ldots & 0 & 0 \\
\vdots & \vdots & \vdots & \vdots & \vdots & & \vdots & \vdots \\
\beta+\alpha y & \alpha \beta & 0 & 0 & 0 & \ldots & \beta & y+\alpha \beta
\end{array}\right]
$$

where $\beta=p^{2}-h^{2}$ and $y=4 p^{2}+2 h^{2}$. Now, we see that the matrix $A$ can be written as the product of matrices $B$ and $C$ where

$$
B=\left[\begin{array}{cccccc}
\alpha & 0 & 0 & \ldots & 0 & 1 \\
1 & \alpha & 0 & \ldots & 0 & 0 \\
0 & 1 & \alpha & \ldots & 0 & 0 \\
\vdots & & & & & \\
0 & 0 & 0 & \ldots & \alpha & 0 \\
0 & 0 & 0 & & 1 & \alpha
\end{array}\right]
$$


and

$$
C=\left[\begin{array}{ccccccc}
p^{2}-h^{2} & 4 p^{2}+2 h^{2} & p^{2}-h^{2} & 0 & \ldots & 0 & 0 \\
0 & p^{2}-h^{2} & 4 p^{2}+2 h^{2} & p^{2}-h^{2} & \ldots & 0 & 0 \\
\vdots & & & & & \\
4 p^{2}+2 h^{2} & p^{2}-h^{2} & 0 & 0 & \ldots & 0 & p^{2}-h^{2}
\end{array}\right]
$$

The system of equations (2.8) has a unique solution if the matrix $A$ is non-singular. We observe that matrix $A$ is non-singular if and only if matrices $B$ and $C$ are non-singular. Obviously, a permuted form of the matrix $C$ is diagonally dominant for any $p>h$. The matrix $C$ is therefore, non-singular. On the other hand, we see that $\operatorname{det} B=\alpha^{n}+(-1)^{n-1}$ which vanishes only when $\alpha=1$ and $n$ is even. Thus we find that the matrix $A$ is non-singular for all non-negative values of $\alpha$ except the particular case when $\alpha=1$ and $n$ is even.

We have thus proved the following:

Theorem 2.1. Given a 1-periodic discrete function $f$ defined over $[0,1]_{h}$ and a uniform sequence of points $P$ in $[0,1]_{h}$, such that $p>h$, there exists $a$ unique 1-periodic discrete cubic spline $s \in \mathscr{S}(4, P, h)$ satisfying the interpolatory condition (2.2) for all non-negative values of $\alpha$ except the case when $\alpha=1$ and $n$ is even.

\section{§3. Error Estimates}

In this section we aim to obtain the bounds of the error function $e=s-f$ for the discrete cubic interpolatory spline $s$ of Theorem 2.1. We estimate the error-bounds over the discrete interval $[0,1]_{h}$, in terms of 'discrete norm' and 'discrete modulus of smoothness'. These will be denoted as $\|g\|$ and $w(g, t)$ for some discrete function $g$ (when there is no scope of confusion in using the same symbols as those used in case of a continuous function).

Discrete norm of a function $g$ in $[a, b]_{h}$ is given by

$$
\|g\|=\max \left\{|g(x)|: x \in[a, b]_{h}\right\} .
$$

For the points $x_{i} \in P$ we shall consider vectors $\left(g\left(x_{1}\right), g\left(x_{2}\right), \ldots, g\left(x_{n}\right)\right)$. We will be using sup norm for vectors defined as

$$
\left\|g_{i}\right\|=\max \left\{\left|g\left(x_{i}\right)\right|, i=1,2, \ldots, n\right\},
$$

and the induced row-max norm for matrices defined as

$$
\|B\|=\max _{1 \leq i \leq n} \sum_{j=1}^{n}\left|b_{i j}\right| \quad \text { where } B=\left(b_{i j}\right)_{n \times n} .
$$

Discrete modulus of smoothness is defined, for a non-negative number $t$, by

$$
w(g, t)=\max \left\{|g(x)-g(y)|:|x-y|<t, x, y \in[a, b]_{h}\right\}
$$


We shall need certain normalized version of usual forward differences, called 'forward-difference-operators'. The $j$-th forward difference operator of a function $g$, denoted by $D_{h}^{(j)} g$ or $g^{(j)}$ is given by

$$
D_{h}^{(j)} g(x)=\left(1 / h^{j}\right) \sum_{i=0}^{j}(-1)^{j-i}\left(\begin{array}{l}
j \\
i
\end{array}\right) g(x+i h) .
$$

Thus in particular,

$$
g^{(1)}(x)=[g(x+h)-g(x)] / h .
$$

Also, for given points $t_{i}, i=1,2, \ldots ;\left[t_{i}, t_{i+1}, \ldots, t_{i+k}\right] g$ denotes the $k$-th divided difference of the function $g$ at the points $t_{i}, t_{i+1}, \ldots, t_{i+k}$. Clearly we have

$$
\begin{aligned}
& g^{\{1\}}(x)=[x-h, x+h] g, \\
& g^{\{2\}}(x)=2[x-h, x, x+h] g,
\end{aligned}
$$

and so on.

In order to obtain error-estimates over a discrete interval we need following Lemma due to Lyche (7):

Lemma 3.1. Let $\left\{a_{i}\right\}_{i=1}^{m}$ and $\left\{b_{i}\right\}_{i=1}^{r}$ be two sequences of non-negative real numbers such that $\sum_{i=1}^{m} a_{i}=\sum_{i=1}^{r} b_{i}$. Suppose $\left\{y_{i j}\right\}_{i=1, j=0}^{m, k},\left\{z_{i j}\right\}_{i=1, j=0}^{r, k} \subset[\alpha, \beta]_{h}$ for some $\alpha, \beta$. Then, for a discrete function $g$ defined on $[\alpha, \beta]_{h}$

$$
\begin{aligned}
& \left|\sum_{i=1}^{m} a_{i}\left[y_{i 0}, y_{i 1}, \ldots, y_{i k}\right] g-\sum_{i=1}^{r} b_{i}\left[z_{i 0}, z_{i 1}, \ldots, z_{i k}\right] g\right| \\
& \quad \leq(1 / k !)\left(\sum_{i=1}^{m} a_{i}\right) w\left(g^{(k)},|I|\right)
\end{aligned}
$$

where $|I|=\beta-\alpha-k h$.

The proof of the Lemma is easily deduced.

We are now set to prove the following:

Theorem 3.2. Let $f$ be a 1-periodic discrete function defined on $[0,1]_{h}$ and $s \in \mathscr{S}(4, P, h)$ be the unique 1-periodic discrete cubic spline satisfying (2.2) for non-negative number $\alpha$ specified by Theorem 2.1, then

$$
\left\|e_{i}^{\{2\}}\right\| \leq K w\left(f^{(2)}, p\right)
$$

and

$$
\left\|e^{\{2\}}\right\| \leq(1+K) w\left(f^{(2)}, p\right)
$$


where constant $K$ is bounded as follows

$$
\begin{aligned}
K & \leq 12 \frac{(1+\alpha)}{|1-\alpha|}, \quad \text { if } n \text { is even and } \alpha \neq 1 ; \\
& \leq 12 \frac{(1+\alpha)}{1+\alpha^{n}} \cdot \frac{1-\alpha^{n}}{1-\alpha}, \quad \text { if } n \text { is odd and } \alpha \neq 1 ; \\
& \leq 6(1+\alpha) \cdot n \quad \text { if } n \text { is odd and } \alpha=1 .
\end{aligned}
$$

Proof of the Theorem. In the system of equations (2.8) we replace $M_{i}$ by $e_{i}^{\{2\}}+f_{i}^{\{2\}}$ and we get

$$
\begin{aligned}
\left(p^{2}-\right. & \left.h^{2}\right) e_{i-1}^{\{2\}}+\left[4 p^{2}+2 h^{2}+\alpha\left(p^{2}-h^{2}\right)\right] e_{i}^{\{2\}} \\
& +\left[p^{2}-h^{2}+\alpha\left(4 p^{2}+2 h^{2}\right)\right] e_{i+1}^{\{2\}}+\alpha\left(p^{2}-h^{2}\right) e_{i+2}^{\{2\}} \\
= & 12 p^{2}\left[x_{i-1}, x_{i}, x_{i+1}\right] f-\left(p^{2}-h^{2}\right) f_{i-1}^{\{2\}}-\left(4 p^{2}+2 h^{2}\right) f_{i}^{\{2\}} \\
& \quad-\left(p^{2}-h^{2}\right) f_{i+1}^{\{2}+\alpha\left[12 p^{2}\left[x_{i}, x_{i+1}, x_{i+2}\right] f-\left(p^{2}-h^{2}\right) f_{i}^{\{2\}}\right. \\
& \left.\quad-\left(4 p^{2}+2 h^{2}\right) f_{i+1}^{\{2\}}-\left(p^{2}-h^{2}\right) f_{i+2}^{\{2\}}\right] \\
= & G_{i} \text { say } ; \quad i=1,2, \ldots, n .
\end{aligned}
$$

Now the system of equation (3.3) can be written as

$$
A E=G \quad \text { or } \quad E=C^{-1} B^{-1} G,
$$

with $E={ }^{t}\left(e_{1}^{\{2\}}, e_{2}^{\{2\}}, \ldots, e_{n}^{\{2\}}\right), G={ }^{t}\left(G_{1}, G_{2}, \ldots, G_{n}\right)$ and $A=B C$ where $B$ and $C$ are given by (2.9) and (2.10) respectively.

Considering the row-max norm we have

$$
\|E\|=\max _{1 \leq i \leq n}\left|e_{i}^{\{2\}}\right| \leq\left\|B^{-1}\right\| \cdot\left\|C^{-1}\right\| \cdot\|G\| .
$$

We observe, in view of Lemma 3.1, that

$$
\left|G_{i}\right| \leq 24(1+\alpha) p^{2} w\left(f^{(2)}, p\right), \quad i=1,2, \ldots, n .
$$

Hence,

$$
\|G\| \leq 24(1+\alpha) p^{2} w\left(f^{(2)}, p\right) .
$$

Also, the row-max norm of $C^{-1}$ is bounded as follows:

$$
\begin{aligned}
\left\|C^{-1}\right\| & \leq\left\{\min _{1 \leq i \leq n}\left(\left|c_{i, i+1}\right|-\sum_{\substack{j \neq i+1 \\
j=1}}^{n}\left|c_{i j}\right|\right)\right\}^{-1} \\
& \leq 1 / 2\left(p^{2}+2 h^{2}\right)
\end{aligned}
$$

[In the above relation the suffix $j=n+1$ is identified with suffix $j=1$, since $C$ is a circulant matrix.] 
Now we find that

$$
B^{-1}=\left\{\frac{1}{\alpha^{n}+(-1)^{n-1}}\right\} \cdot\left[\begin{array}{ccccc}
\alpha^{n-1} & (-1)^{n-1} & (-1)^{n-2} \alpha & \ldots & -\alpha^{n-2} \\
-\alpha^{n-2} & \alpha^{n-1} & (-1)^{n-1} & \ldots & \alpha^{n-3} \\
\vdots & \vdots & \vdots & & \vdots \\
(-1)^{n-1} & (-1)^{n-2} \alpha & (-1)^{n-3} \alpha^{2} & \ldots & \alpha^{n-1}
\end{array}\right]
$$

Clearly the row-max norm of $B^{-1}$ is

$$
\begin{aligned}
& =\frac{1}{|1-\alpha|}, \quad \text { if } n \text { is even and } \alpha \neq 1 ; \\
& =\frac{1}{1+\alpha^{n}} \cdot \frac{1-\alpha^{n}}{1-\alpha}, \quad \text { if } n \text { is odd and } \alpha \neq 1 ; \\
& =n / 2, \quad \text { if } n \text { is odd and } \alpha=1 .
\end{aligned}
$$

Therefore estimates (3.5)-(3.7) when combined with (3.4) yield (3.1).

Next, we see that $s^{\{2\}}$ is linear in $\left[x_{i-1}, x_{i}\right] i=1,2, \ldots, n$ and is given by (2.3). Therefore

$$
\begin{aligned}
p e^{\{2\}}(x)= & \left(x_{i}-x\right)\left(M_{i-1}-f_{i-1}^{\{2\}}\right)+\left(x-x_{i-1}\right)\left(M_{i}-f_{i}^{\{2\}}\right) \\
& +\left(x_{i}-x\right)\left(f_{i-1}^{\{2\}}-f^{\{2\}}(x)\right)+\left(x-x_{i-1}\right)\left(f_{i-1}^{\{2\}}-f^{\{2\}}(x)\right) .
\end{aligned}
$$

Thus

$$
\left|e^{\{2\}}(x)\right| \leq\left\|e_{i}^{\{2\}}\right\|+w\left(f^{(2)}, p\right) .
$$

In view of (3.1) and (3.8) we get (3.2). This completes the proof of the Theorem 3.2 .

Discussion. In the case $\alpha \neq 1$, we observe from (3.2) that as $n$ increases to infinity, $\left\|e^{\{2\}}(x)\right\|$ goes to zero. Thus $s^{\{2\}}$ converges uniformly to $f^{\{2\}}$ as $n \rightarrow \infty$ in this case. On the other hand, when $\alpha=1$ and $n$ is odd, error $\left\|e^{\{2\}}\right\|$ varies as $\left\|f^{(2)}\right\|$. Thus the rate of convergence of $s^{\{2\}}$ to $f^{\{2\}}$ is fast except in the case when $\alpha=1$ and $n$ is odd.

\section{§4. Remarks}

4.1. Theorem 2.1 and Theorem 3.2 are more general than the corresponding results of Lyche [7] where he considers a discrete cubic spline satisfying condition (2.2) for the case $\alpha=0$ only.

4.2. When $h \rightarrow 0$, results of Theorems 2.1 and 3.2 correspond to the results of Theorems 1 and 4 of [3] for usual cubic splines. But wider range for weight $\alpha$ and sharper error estimates than those obtained in [3] are established in Theorems 2.1 and 3.2. 
4.3. It is easy to observe from (2.2)-(2.7) that when $\alpha=1$ and $n$ is odd, the discrete cubic spline interpolant of Theorem 2.1 matches the functionvalues at the meshpoints, i.e. we have

$$
s\left(x_{i}\right)=f\left(x_{i}\right), \quad i=1,2, \ldots, n,
$$

in this case.

\section{Acknowledgement}

The author would like to thank Dr. K. C. Deomurari for certain useful suggestions.

\section{References}

[1] Ahlberg, J. H., Nilson, E. N. and Walsh, J. L., The Theory of Splines and Their Applications, Academic Press, New York, 1967.

[2] Astor, P. H. and Duris, C. S., Discrete L-Splines, Numer. Math., 22 (1974), 393-402.

[3] Chatterjee, A. and Dikshit, H. P., On error bounds for cubic spline interpolation, J.O. Math. Soc., 1 (1982), 1-11.

[4] Dikshit, H. P. and Powar, P. L., Discrete cubic spline interpolation, Numer. Math., 40 (1982), 71-78.

[5] —, Area matching interpolation by discrete cubic splines, Approx. Theory Appl. Res. Notes Maths., 133 (1985), 34-45.

[6] Lyche, T., Discrete polynomial spline approximation methods, Report RRI 2, University of Oslo (1975).

[7] - Discrete cubic spline interpolation, Report RRI 5, University of Oslo, (1975).

[8] Mangasarian, O. L. and Schumaker, L. L., Discrete splines via mathematical programming, SIAM J. Control. 9 (1971), 174-183.

[9] Schumaker, L. L., Constructive aspect of discrete polynomial spline functions, Approximation Theory II, Academic Press, New York, (1976), 469-476.

[10] —, Spline Functions, Basic Theory, John Wiley and Sons, New York, 1981. 